\title{
Wie schreibt man Universitätsgeschichte?
}

\author{
Marian Füssel
}

Die Universitätsgeschichtsschreibung kann auf eine lange Tradition verweisen, hat bereits diverse Paradigmenwechsel hinter sich und ist auf vielfältige Weise national wie international institutionalisiert (Paletschek 2011). Dennoch scheint sie im Vergleich zur Wissenschafts- und in jüngerer Zeit zur Wissensgeschichte immer noch einen unsicheren Status zu genießen. Zumindest dürfte ein klares Bekenntnis zu den letztgenannten Feldern den meisten, gerade jüngeren Wissenschaftlerinnen und Wissenschaftlern leichter fallen. $\mathrm{Ob}$ eine strikte Trennung in Universitäts- und Wissenschaftsgeschichte überhaupt möglich und sinnvoll ist, kann allerdings mit Recht bezweifelt werden. Die fragile Situation der Universitätsgeschichtsschreibung ist sicher $\mathrm{zu}$ weiten Teilen dem vielzitierten Spannungsverhältnis von „Jubiläumsschrift und Alltagsarbeit“ (Hammerstein 1983) geschuldet, das Universitätsgeschichte in Zeiten großer Jubiläen lokal und temporär zu einer geschätzten und vielgelobten Tätigkeit macht. Doch sobald die Jubiläen vorbei sind, schwindet das Interesse ebenso rasch wie es gekommen ist. Ja, als Universitätshistoriker gerät man eventuell gar in den Ruf, selbstreferentielle Orchideenforschung zu betreiben, die nur um die Vorgeschichte der eigenen akademischen Lebenswelt kreist, die vermeintlich wirklich wichtigen Dinge aber außer Acht lässt. So kommt es, dass Universitätsgeschichtsschreibung in der Regel nur ein thematisches Standbein einer Forscherpersönlichkeit ist. Ein Faktum, das in sich nicht weiter tragisch, vielleicht für die eigene Arbeit sogar recht heilsam ist, weil es Scheuklappendenken und -forschen erschwert. Problematischer scheint mir hingegen für die Universitätsgeschichtsschreibung als Subdisziplin der Geschichtswissenschaft und der Wissenschaftsgeschichte, dass diese Teilzeit-, Ad-hoc- und Auftragsarbeit es offenbar schwierig macht, eigene und als solche auch anerkannte, das heißt konsensfähige epochenübergreifende Methoden, Zugänge, Theorien und Narrative $\mathrm{zu}$ entwickeln. So hat die 
Universitätsgeschichte zwar ihre eigene Epochennomenklatur entworfen, die mit Peter Moraw die Entwicklung in eine vorklassische, klassische und nachklassische Phase unterteilt, sich damit jedoch weder in die Diskussion um Epochenzuschnitte einbringen, noch verbindlich in Handbuchliteratur etablieren können, zumal der inzwischen verstorbene Doyen der deutschen Universitätsgeschichtsschreibung sie später selbst wieder relativiert hat.

Es mag sich vielfach zudem der Eindruck einstellen, die Universitätsgeschichtsschreibung arbeite sich als eine Art notorischer Nachzügler immer an den Themen ab, welche die allgemeine Geschichtswissenschaft gerade vorgibt. Viele die historische Forschung prägende Themen - hier mit einem Fokus auf der Vormoderne - wie etwa Staatsbildung, Konfessionalisierung und Sozialdisziplinierung, Memoria und Stiftungswesen, Hexenverfolgung, Devianz, Migration und Kulturtransfer, Geschlechterordnungen, symbolische Kommunikation oder materielle Kultur, haben der Universitätsgeschichte wichtige Impulse verliehen - doch auch ihrerseits aus der Universitätsgeschichte auf die allgemeine Geschichte gewirkt. Auch sollte man nicht Inhalt und Methode verwechseln. Die paradigmatische Abfolge der tonangebenden methodischen Ansätze verlief tatsächlich weitgehend parallel. Nach Anfängen der Verwissenschaftlichung um 1800 (Meiners 1802-1804) folgten im 19. Jahrhundert große, an der Verfassungsgeschichte orientierte Gesamtdarstellungen (Denifle 1885; Kaufmann 1888/1896; Rashdall 1895), flankiert von zahlreichen grundlegenden Quelleneditionen, von denen die Forschung noch heute zehrt. Um 1900 kamen cultur- und sittenhistorische Abhandlungen hinzu, und die Universitätsgeschichte trat in eine Phase der Spezialisierung ein. Bereits die 1904/1905 erschienene monumentale Bibliographie zur Universitätsgeschichte von Wilhelm Erman und Ewald Horn legt davon beredt Zeugnis ab. Nach dem Zweiten Weltkrieg traten hochschulsoziologische Zugänge auf den Plan (Helmut Schelsky, Pierre Bourdieu, Rudolf Stichweh), die zusammen mit quantifizierenden historischen Ansätzen ab den 1970er und 1980er Jahren eine sozialhistorische Wende der Universitätsgeschichtsschreibung einleiteten. In den 1990er Jahren folgten schließlich kulturhistorische Ansätze und Einflüsse aus der neuen französischen und angloamerikanischen Wissenschaftsgeschichte. Die Abfolge dieser Paradigmen ist jedoch nicht konsekutiv zu verstehen, sondern hat vielmehr zu einer Ausdifferenzierung der Universitätshistoriographie geführt, die heute - zumindest epistemologisch problemlos quantifizierende mit qualitativen Analysen zu verbinden weiß. So ist die sozialhistorische Matrikelforschung aus der Hochschulforschung nicht mehr wegzudenken und auch für die allgemeine Geschichte zu einem Referenzpunkt für quantifizierende Zugänge gerade zur vormodernen europäischen Geschichte geworden.

Eine besondere Problematik gegenwärtiger Universitätsgeschichtsschreibung resultiert allerdings aus den Prozessen ihrer internen Ausdifferenzierung und Departementalisierung. So kam es beispielsweise zu einer Trennung von 
Wissenschafts- und Universitätsgeschichte sowie von Studenten- und Universitätsgeschichte, die sich auch in der Existenz unterschiedlicher Zeitschriften artikuliert und institutionalisiert. Ohne einer histoire totale das Wort zu reden, sollten diese Trennungen jedoch nach Möglichkeit überwunden werden, oder zumindest der individuellen Forschungsarbeit keine Grenzen setzen. Trennt man Wissenschafts- und Universitätsgeschichte, so dichotomisiert man zwischen „science“ und „context", anstatt „science in context" zu erforschen. Gerade der institutionelle Rahmen der Universität eröffnet die Chance, die Trennung in eine Wissenschaftsgeschichte der Naturwissenschaften und eine Wissenschaftsgeschichte der Geistes- und Kulturwissenschaften zu unterlaufen und deren Differenzierung überhaupt erst historisch sichtbar zu machen. Die Verknüpfung von inhaltlicher und institutioneller Dimension, deren Trennung eigentlich eine rein analytische ist, konfrontiert jede einzelne Forscherin und jeden einzelnen Forscher jedoch auch unweigerlich mit den Grenzen individueller fachlicher Kompetenzen. Akademische Doppelsozialisationen beispielsweise in Physik und Geschichtswissenschaft oder Soziologie und Biologie können hilfreich sein, dürfen aber nicht zur Voraussetzung von historischer Universitätsforschung gemacht werden. Insofern kann die disziplinäre Arbeitsteilung immer auch als Ausdruck von Professionalisierung interpretiert und der Ruf nach Transdisziplinarität als ein latenter Aufruf zum Dilettieren gedeutet werden. Vielleicht muss zunächst der interdisziplinäre Dialog hier eine Verständigung über die jeweiligen disziplinären Denkstile und Praktiken befördern, bevor eine Aufhebung der Grenzen projektiert werden kann.

Trennt man Studenten- und Universitätsgeschichte, überlässt man zumindest im deutschsprachigen Raum das Feld der Studentengeschichte nicht nur weitgehend einer ideologisch fragwürdigen Selbstvergewisserungshistoriographie von Studentenkorporationen, sondern verliert auch einen signifikanten Bestandteil der Universitäten aus den Augen. Studenten werden allzu leicht entweder auf statistisches Material, Hörerzahlen und Frequenzen oder den wissenschaftsfernen Eigensinn ihrer jugendkulturellen Lebenswelten reduziert. Die Universität als Organisation bildet jedoch eine Schnittstelle zwischen dem Wissenschafts- und dem Erziehungssystem einer Gesellschaft. Der Faktor der Bildung sollte gegenüber dem der Wissensproduktion daher nicht zu gering gewichtet werden. So stellt die Geschichte des akademischen Unterrichts über viele Disziplinen und Zeiträume hinweg nach wie vor noch ein großes Desiderat dar.

Die Frage nach dem „wie“ von Universitätsgeschichtsschreibung lässt sich allerdings nicht mit einzelnen Desideratlisten beantworten, sondern muss tiefer ansetzen. Dazu bedarf es einer kurzen Standortbestimmung. Mein eigener Zugang zur Universitätsgeschichte lässt sich immer noch am ehesten als ein kulturwissenschaftlicher, von historisch-anthropologischen Ansätzen geleiteter beschreiben. Dies kann trotz oder vielleicht sogar gerade aufgrund der 
Tatsache, dass die Kulturgeschichte im deutschsprachigen Raum mittlerweile den historiographischen Mainstream darstellt, immer noch zu einem grundlegenden Missverständnis führen. Eine Historische Anthropologie der Universität ist weder auf einen Gegenstand noch eine spezifische Methode fokussiert, sondern verfolgt eine bestimmte Perspektive und Heuristik, aus der heraus jedes Thema beforscht werden kann. In meiner Arbeit zur symbolischen Praxis der akademischen Gelehrtenkultur der Frühen Neuzeit fiel allerdings beides zusammen: ein Gegenstand, der bereits Thema der eher vom Forschungsobjekt her definierten älteren Culturgeschichte hätte sein können und teils auch war, und die Methoden und Theorien der Neuen Kulturgeschichte und Historischen Anthropologie (Füssel 2006). Eine historischanthropologische Perspektive in der Universitätsgeschichtsschreibung bedeutet jedoch gerade nicht, der Palette der Themenfelder nun einfach Themen wie Festkultur, Habitusformierung und symbolische Ordnungen hinzuzufügen, sondern vielmehr an einer grundlegenden Historisierung der Universitäten zu arbeiten: Historisierung verstanden im Sinne von Karl Marx ebenso wie von Johann Gustav Droysen, Friedrich Nietzsche sowie Michel Foucault. Am Beispiel der symbolischen Praktiken bedeutet Historisierung konkret nicht ein belächelndes Abhaken überwundener vormoderner Alterität, sondern Schärfung des Bewusstseins für die Rationalität symbolischer Kommunikation und Sensibilisierung für die Beobachtung von Elementen ständischer Vergesellschaftung auch in unserer modernen akademischen Lebenswelt.

Der Begriff der Kulturgeschichte hat seinerseits inzwischen schon so viel Unschärfe erlangt und ist mittlerweile selbst Gegenstand der Historisierung, dass er hier nicht erneut als Kampfbegriff ins Feld geführt werden soll; die wesentlichen Schlachten sind ohnehin bereits geschlagen. Es kann also nicht darum gehen, die Frage nach dem „wie“ der Universitätsgeschichtsschreibung mit einem sukzessiven Abklappern bestehender oder dem Abwarten noch kommender cultural turns zu beantworten, obwohl das im Einzelnen durchaus innovative Ergebnisse zeitigen kann. So wurden etwa linguistic, iconic, spatial, postcolonial oder material turn, wenn auch begrenzt, so doch zum Teil heuristisch gewinnbringend in der Universitätsgeschichtsschreibung zur Anwendung gebracht. Wenn etwas aus der kulturwissenschaftlichen Wende bleiben wird, ist es vielmehr der Gedanke der radikalen Historisierung (Breidbach 2011).

Radikale Historisierung ist das Grundanliegen jeder historischen Forschung, ließe sich einwenden, was soll daran nun für die Universitätsgeschichtsschreibung neu oder besonders hilfreich sein? Das ist zweifellos richtig, doch wird der Aufruf zur Historisierung immer noch zu selten wirklich ernst genommen und empirisch umgesetzt. Und damit sind nicht nur die traditionsbekräftigenden und identifikatorischen Ansätze mancher Jubiläumsschriften gemeint. Gerade die scheinbare Vertrautheit mit der 
Institution, aus der heraus die meisten Universitätshistorikerinnen und Universitätshistoriker die Hochschulen erforschen, kann ein massives Hindernis für deren Historisierung darstellen. Begriffe wie Professor, Senat, Dekan, Promotion, Habilitation, Berufungsverfahren, Vorlesung, Labor etc. scheinen sich wie von selbst $\mathrm{zu}$ verstehen und eine überzeitliche Vergleichbarkeit zu suggerieren. Jede einzelne Rolle, jedes Amt, jedes Gremium, jede materielle Infrastruktur und jedes Verfahren hat jedoch seine eigene Geschichte und bei näherem Hinsehen - etwa am Beispiel der Habilitation - erweist sie sich sogar noch als weitgehend ungeschrieben. Anstatt den Selbstverständlichkeiten unserer Rationalisierungs- und Modernisierungsnarrative einfach zu folgen, gilt es vielmehr, diese konsequent zu hinterfragen. So kann sich die heuristische Fiktion des ethnologischen Blicks auf die eigene Kultur immer wieder als produktiv erweisen, indem sie Unbekanntes oder Vergessenes sichtbar macht oder Altbekanntes in neues Licht rückt.

Im Hinblick auf unsere Leitfrage sind zwei weitere strukturelle Hindernisse der Historisierung der Universitäten anzusprechen. Das ist zum einen die Spannung zwischen der Geschichte jeder individuellen Universität und ihren Akteuren, die aus lokaler Perspektive als historisch einzigartig erscheint, und den allgemeinen strukturellen Entwicklungen. So hat Rainer Christoph Schwinges einmal das komparatistische Postulat erhoben, „daß man eine Universität erst dann richtig kennt, wenn man sie alle kennt" (Schwinges 1986: 10). Das macht gewiss viel Arbeit und zielt jenseits des Spätmittelalters wohl auch nicht auf empirische Vollständigkeit, ist aber unabdingbar, um Verzeichnungen und Individualitätsfiktionen in der Geschichte jeder einzelnen Universität zu vermeiden. Zum anderen ist der Historisierung die für die Wissenschaftsgeschichte allgemein geltende Versuchung einer linearen Fortschritts- oder Niedergangserzählung hinderlich, in der Wissen immer wahrer oder die Studierenden immer dümmer werden. Eine moderne, vergleichend vorgehende Universitätsgeschichte arbeitet vielmehr an einer stetigen Historisierung der Universität, die auch abweichende, fremde oder unverständliche Phänomene ernst nimmt, ohne sie in das Korsett modernisierungstheoretischer Formeln zu zwängen. Die Geschichte einer Universität und ihrer Angehörigen ist nicht deshalb bedeutsam, weil hier vermeintlich etwas zum ersten Mal passierte und man so der Idee des Ursprungs huldigen kann, sondern vielmehr als Teil einer Serie. Damit ist ein allgemeines historiographisches Problem berührt, das man als die Austreibung der großen Erzählungen und Modernisierungstheorien aus der historischen Kulturwissenschaft beschreiben kann. Jenseits der Frage, ob das viel beschworene Ende der Meisterzählungen nicht selbst eine Meisterzählung ist, gehört es mittlerweile zum guten Ton sich von größeren Verlaufsmodellen und Narrativen über mehrere Jahrhunderte hinweg zu distanzieren, ebenso wie Theorien von Rationalisierung, Zivilisierung, Disziplinierung, Ausdifferenzierung, Professionalisierung, Ökonomisierung etc. zumindest kritisch zu 
hinterfragen. Diese Erzählmuster hatten jedoch einige entscheidende Vorteile. Sie ermöglichen die Reduktion von Komplexität und stellten damit die Bedingung der Möglichkeit jeder Synthetisierung eines stetig wachsenden historischen Wissens bereit. Sie dienten zudem als Ausweis von historischer Relevanz, was vor allem für die sich mit den vormodernen Jahrhunderten beschäftigenden Historikerinnen und Historiker zum Teil existenzsichernde Bedeutung trägt. Deutlich wurde das etwa, als vor einigen Jahren jede Teilepoche auf einmal, ihren' Weg in die Wissensgesellschaft fand und damit zur Selbstaufwertung ansetzte. So setze neben der Geschichte des 19. Jahrhunderts beispielsweise auch die Frühneuzeitforschung und die Mediävistik dazu an, die soziologische Gegenwartsbeschreibung der seit den späten 1960er Jahren verorteten Wissensgesellschaft mit immer längeren historischen Genealogien zu versehen (Füssel 2007).

Dass Mikrogeschichte, Praxeologie und Modernisierungstheorie jedoch kein Widerspruch sein müssen, hat William Clark mit seinem Buch Academic Charisma and the Origins of the Research University gezeigt (Clark 2006). Damit ist Clark das seit vielen Jahren weltweit wohl meistdiskutierte Werk zur frühneuzeitlichen, vorwiegend deutschen Universitätsgeschichte gelungen, das eben so viel Lob wie Kritik geerntet hat. So löste die mit kalifornischer Ironie vorgetragene Verbindung von einer an Max Weber und Michel Foucault orientierten Makrooptik mit minutiösen Mikrostudien zu den „little tools of knowledge“ (Clark 2006: 6) wie Vorlesungsverzeichnissen, Visitationsberichten oder Prüfungsverfahren auch manche Irritation aus. Irritierende Bücher sind es aber, die unsere Diskussion weiterbringen; irritierend an manchen Jubiläumsschriften ist jedoch vielfach wohl eher ihr enormer Umfang - zum Teil vielleicht dem Versuch der Umrechnung von beanspruchter Bedeutung in Seitenzahlen geschuldet - als ihr Inhalt. Doch es wäre billig, dieses anlassgebundene Schrifttum zum Popanz mangelnder Innovation zu machen. Vielen Jubiläen verdanken wir moderne Klassiker der Universitätsgeschichtsschreibung wie in narrativer Hinsicht etwa Eine deutsche Universität von Walter Jens, ein Werk das 1977 anlässlich des 500jährigen Gründungsjubiläums der Universität Tübingen erschien. Damit wären wir schließlich bei der Frage des „wie“ in seiner ganz konkreten, erzählerischen Schreibart. Auch hier sind ähnliche Herausforderungen gegeben wie für jede andere Geschichtsschreibung auch, und es wäre vielleicht eine reizvolle Aufgabe im Stile Hayden Whites einmal nach den narrativen Modellierungen der Metahistory der Universitätsgeschichtsschreibung zu fragen. So folgen neben den mutmaßlichen Tragödien untergegangener Hochschulen wie Helmstedt oder Altdorf viele Universitätsgeschichten scheinbar dem Modell des klassischen Bildungsromans: Auf eine frühe Phase der Behauptung in der Umwelt folgen verschiedene Krisen, nach deren Überwindung der Protagonist 
ein anerkanntes Mitglied des Wissenschaftssystems geworden ist, das selbst in der Lage ist, andere zu bilden. Eine historisierende Universitätsgeschichtsschreibung sollte sich dieser „emplotments“ zumindest bewusst sein, ganz verlassen können wird auch sie die Metanarrative ohnehin nicht.

\section{Literatur}

Breidbach, Olaf 2011. Radikale Historisierung. Kulturelle Selbstversicherung im Postdarwinismus. Berlin: Suhrkamp.

Denifle, Heinrich 1885. Die Universitäten des Mittelalters bis 1400, Bd. 1. Die Entstehung der Universitäten des Mittelalters bis 1400. Berlin: Weidmann.

Clark, William 2006. Academic Charisma and the Origins of the Research University. Chicago: University of Chicago Press.

Füssel, Marian 2006. Gelehrtenkultur als symbolische Praxis. Rang, Ritual und Konflikt an der Universität der Frühen Neuzeit. Darmstadt: Wissenschaftliche Buchgesellschaft.

Füssel, Marian 2007. Auf dem Weg zur Wissensgesellschaft. Neue Forschungen zur Kultur des Wissens in der Frühen Neuzeit. Zeitschrift für historische Forschung (34): 273-289.

Hammerstein, Notker 1983. Jubiläumsschrift und Alltagsarbeit. Tendenzen bildungsgeschichtlicher Literatur. Historische Zeitschrift (236): 601-633.

Kaufmann, Georg 1888/1896. Geschichte der deutschen Universitäten, 2 Bde. Stuttgart: Cotta.

Meiners, Christoph 1802-1804. Geschichte der Entstehung und Entwicklung der hohen Schulen unseres Erdteils, 4 Bde. Göttingen: Johann Friedrich Röwer (ND Aalen: Scientia-Verlag 1973).

Paletschek, Sylvia 2011. Stand und Perspektiven der neueren Universitätsgeschichte. NTM. Zeitschrift für Geschichte der Wissenschaften, Technik und Medizin (N. F. 19): 169-189.

Rashdall, Hastings 1895. The Universities of Europe in the Middle Ages. Oxford: Clarendon (Neuberarbeitung von Frederick M. Powicke und A. B. Emden, 3 Bde. 1936. Oxford: Oxford University Press).

Schwinges, Rainer Christoph 1986. Deutsche Universitätsbesucher im 14. und 15. Jahrhundert. Studien zur Sozialgeschichte des Alten Reiches. Stuttgart; Franz Steiner Verlag.

\section{Marian Füssel}

Seminar für Mittlere und Neuere Geschichte

Georg-August-Universität Göttingen,

Kulturwissenschaftliches Zentrum

Heinrich Düker Weg 14

37073 Göttingen

Deutschland

E-Mail: Marian.Fuessel@phil.uni-goettingen.de 